Q18 CEF"-centro brasileiro de pesquisas físicas Notas de Física

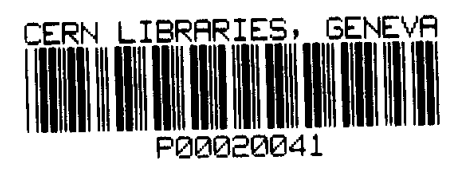

$$
C B P F-N F-006 / 93
$$

An Effective Liquid Drop

Description for the Exotic Decay

of Nuclei

by

Marcello G. Gonçalves and S.B. Duarte 


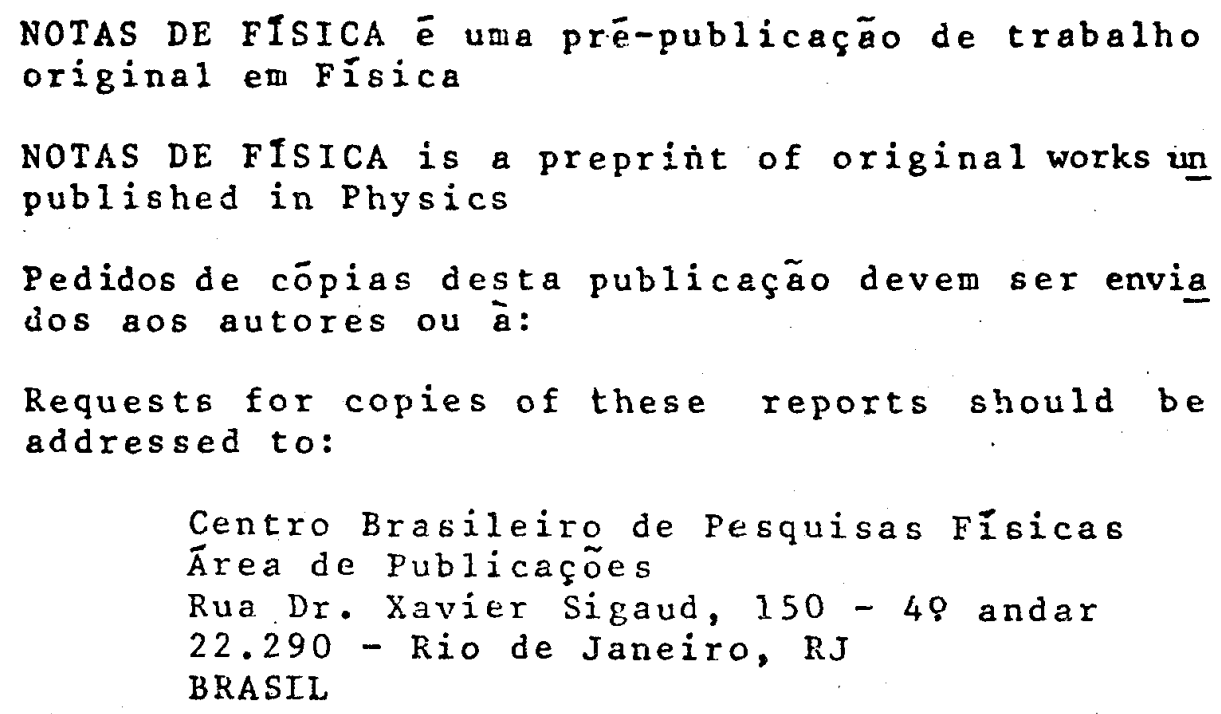


ISSN 0029-3865

$C B P F-N F-006 / 93$

\section{An Effective Liquid Drop \\ Description for the Exotic Decay \\ of Nuclei}

by

Marcello G. Gonçalves' and S.B. Duarte

Centro Brasilciro de Pesquisas Físicas - CBPF/CNPq

Rua Dr. Xavier Sigaud, 150

22290-180 - Rio de Janciro, RJ ... Brasil

'Departamento de Ciencias Naturais

Fundasão de Ensino Superior de Sào João del Rei - FUNREI

36300-000 - São João del Rei, MG - Brasil 
- Abstract

The present model describes the exotic decay of nuclei including the molecular phase of the fragments by using only the basic elements of the liquid drop fission model. The Coulomb potential energy is the exact solution of the Poisson equation for a uniform charge distribution in the nuclear rolume and the surface potential is defined in texms of an effective surface tension. The WernerWheeler sproximation for the velccity field of the nuclear llow determines the inertial coefficient of the reduced onedimensional barricr penetrability problem. Tie model is weil succeedsd to calculate the hali-life of exotic decay process as well as to calculate the ajgha desintegration hali-life.

Key-words: Surface tension; Gamow penetrability; Half life for exotic and alpha decay. 


\section{Introduction}

Although the discovery of the nuclear fission date of the thirties, the fission process with great mass asymmetry was observed only in the seventies. For the first time this exotic decay was observed at Centro Brasileiro de Pesquisas Fiisicas (CBPF), during a work on $U^{238}$ search track fission produts ${ }^{(1.2 .3)}$. Later, other independent experimental observations by Rose and Jones ${ }^{[4]}$ and Alessandrov et. al. ${ }^{[5]}$ confirmed the CBFF pioneering results. Since then, many different models and theoretical estimates have appeared to explain the experimental results and to predict new types of such nuclear processes.

Different nature and forms of the potential has been used in these models $16,7,8,2,10$. The results are led to a reasonable accorclance with the observation by adjusting the model parameters. The maximum deviations are within two units around the logarithm of experimental half-live. The number of parameters employed in each model is a consequence of the nature of the potential barrier used ${ }^{17,8 i}$, or it depends on the adopted way in which the mass and charge vary in the prescission phase ${ }^{(8,11)}$. In addition, it also depends on the empirical method of using the zero point vibrational energy of the system ${ }^{[6,7]}$.

In this work we calculate the half-lives for exotic decays considering a double spherical parametrization for the shape of the deformed nuclear system during the fission process. Although this shape parametrization has been used in others exotic decay models, for the first time we make use of an analytical closed expression to calculate.Coulomb energy of the molecular phase of the process. The multidimensional evolution of the system is reduced to the onedimensional case by the geometrical constraints to preserve the adopted shape in the course of the whole process, and also keeping constant the total volume of the system. In the reduced onedimensional p:oblem the Gamow penetrability factor is calculated using an effective mass, determined by using the Werner-Wheele: approximation for the fuid velocity field of the nuclear flow. To complete a basic element of the liquid drop scheme a surface term is included in the potential of the model, with a convenient definition of the surface tension. With only these minimal ingredients of the fission theory in the context of the liquid drop model, we get results in excelent accordance with experimental data for both exotic and alpha decays.

\section{The Model: Shape Parametrization and Potential}

In the molecular phase of the process the geometrical configuration of the deformed system is approximated by intersecting spheres with different radii. For the complete specification of this configuration it is necessary four independent coordinate, disregarding the location of the center-of-mass of the system. We show in fig.1 a sketch of a generic configuration where we specify our choice of coordirates: the radii of each spherical segment, $R_{1}$ and $R_{2}$; the height of the largest spherical segment, $\xi$, and the distance between their geometric centers, $\zeta$. At the end of the prescission phase the system reaches a limiting configuration of two spherical tangent fragments with radii $\bar{R}_{1}$ and $\bar{R}_{2}$, respectively, for the cluster emitted and the heavier daughter.

To maintain the adopted shape parametrization for the deformed nuclear system, it 
is necessary to establish a geometric constraint,

$$
R_{1}^{2}-(\zeta-\xi)^{2} \div R_{2}^{3}-\xi^{2}
$$

keeping a common contact section of the spherical segments during prescission phase. Also the constant total volume of the systern is consiciered as another constraint relation, which is expresed in our coordinates by

$$
2\left(R_{1}^{3}+R_{2}^{3}\right)+3\left[R_{1}^{2}(\zeta-\xi)+R_{2}^{2} \xi\right]-\left[(\zeta-\xi)^{3}+\xi^{3}\right]=4 R_{p}^{3}
$$

where $R_{p}$ is the parent nuclens radius. During the whole molecular phase of fragments we have taken a constant radius to the spherical segment corresponding to the nascent cluster, i. e., we have fixed $R_{1}=\ddot{R}_{1}$.

The model considers only the Coulomb and surface potential energy contributions to the deformation energy of the systeni. Analytical models for exotic decays have never used before an explicit expression for the coulomb energy during the prescission phase. In the most precise way the Conlomb energy has been taken into account by folding numerically the charge density ${ }^{6,14}$ ! in nuciear volune. In our calculation we have made use of Gaudin expression ${ }^{12}$ for the electrostatic energy of spherical portions of uniform. charge distribution,

$$
V_{C}=\frac{8}{9} \pi a^{5} \varepsilon\left(x_{1}, x_{2}\right) \rho_{c}
$$

where $p_{c}$ is the initial charge density, $\varepsilon$ is a function of angular variables $x_{1}$ and $x_{2}$,

$$
\begin{aligned}
& x_{2}=\pi \cdots \theta_{2} \\
& x_{3}=\theta_{2}-\pi .
\end{aligned}
$$


which are defined in terms of the angles $\theta_{1}$ and $\theta_{2}$, shown in fig.1.

The expression for the $\varepsilon$ factor in terms of auxiliary functions $f$ and $g$ is

$$
\begin{aligned}
\varepsilon\left(x_{1}, x_{2}\right)= & \left(\frac{1}{\sin ^{2} x_{2}}-\frac{1}{\sin ^{2} x_{1}}\right)\left[\frac{f\left(x_{2}\right)}{\sin ^{2} x_{2}}-\frac{f\left(x_{1}\right)}{\sin ^{2} x_{1}}\right]- \\
& \left(\cot x_{2}+\cot x_{1}\right)\left[\frac{f^{\prime}\left(x_{2}\right)+\frac{\pi}{4}}{\sin ^{2} x_{2}}+\frac{f^{\prime}\left(x_{1}\right)+\frac{\pi}{1}}{\sin ^{2} x_{1}}\right]+ \\
& \frac{1}{\sin ^{2} x_{1} \sin ^{2} x_{2}}\left[f\left(x_{1}+x_{2}\right)+\frac{1}{3} \sin ^{2}\left(x_{1}+x_{2}\right)\right]+ \\
& \frac{\pi}{8}\left[g\left(x_{1}\right)+g\left(x_{2}\right)\right]
\end{aligned}
$$

where $f^{\prime}$ is the derivative of $f$ with respect to its argument. Explicitly, the auxiliary functions $f, f^{\prime}$ and $g$ are given by

$$
\begin{aligned}
f(x) & =1-x \cot x-\frac{\pi}{2} \tan \frac{x}{2}, \\
f^{\prime}(x)+\frac{\pi}{4} & =\frac{2 x-\sin (2 x)}{2 \sin ^{2} x}-\tan ^{2} \frac{x}{2}, \\
g(x) & =\left(1.5+\tan ^{2} \frac{x}{2}+3 \tan ^{4} \frac{x}{2}\right) \tan \frac{x}{2}+\frac{2}{\sin ^{3} x},
\end{aligned}
$$

with $x$ assuming the values appearing as arguments of these auxiliary functions in eq. $(x)$. The above expression of coulomb energy is the exact solution of the Poisson equation for unifom charge distribution in the system volumeil ${ }^{i}$.

For the surface potential we lave introduced an effective surface tension, $\sigma_{e} f$, to the 
deformed systern, defined throngh the equation

$$
\frac{3}{5} e^{2}\left(\frac{Z_{p}^{2}}{R_{r}}-\frac{Z_{1}^{2}}{\bar{R}_{1}}-\frac{Z_{2}^{2}}{\bar{R}_{2}}\right)+4 \pi \sigma_{e !}\left(R_{p}^{2}-\bar{R}_{1}^{2}-\bar{R}_{2}^{2}\right)=Q
$$

where $Z_{p}$ and $Z_{i} e(i=1,2)$ are the nuclear charge of the parent nucleus and of the frag. ments, respectively. This definition establishes that the difference between the energies of initial end final configurations of the system reproduces the energy released in the disintegration, $Q=M-M_{1}-M_{2}$. The masses in the Q-value expression were taken from nucleas data table ${ }^{(15)}$. Then, for the surface potencial we have,

$$
V_{1}=\sigma_{s} s\left(S_{1}+S_{2}\right)
$$

with the surface of each spherical segnent,

$$
S_{i}=\pi R_{i}\left(R_{i}+\delta_{i}\right) \text {, }
$$

and

$$
\delta_{i}= \begin{cases}\zeta-\xi & i=1 \\ \zeta & i=2\end{cases}
$$

The effects of the centrifugal potention in the molecular phase cannot be descussed without a cereful analysis of argular momentun transter in the hydrodinamic flow of the nuclear ilud. In a simplifying approximation all models which take into account this term have considered it only after the scission point,

$$
V_{C}=\frac{h^{2} t(\ell+1)}{2 \pi}
$$


In this approximation, the effect of the centrifugal potential to the half-life of exotic decay is completly negligible[16.17). The reduced mass, $\bar{\mu}=M_{1} M_{2} /\left(M_{1}+M_{2}\right)$, defines the rotational inertia of the sytem after scission point.

In fig. 2 it is shown our oncdimensional potential,

$$
V=V_{c}+V_{s}+V_{l}-V_{0}
$$

provide with the constraints given by eqs. $(1,2)$ and with the constant radius of nascent cluster. In the equation above, $V_{0}$ means the reference of potential corresponding to the sim of self potential energies (Coulomb and surface) of each fragment in the asymptotic configuration.

\section{Gamow Factor and Decay Half-Life}

The quantum transition rate from initial to final state of the system has been determined by reducing the problem to the onedimensional barrier penetrability, similarly to Gamow alpha decay theory ${ }^{13\}}$. Even when the emphasis of the model is on the fission aspects of the process, the decay rate calculation uses the same procedure. The peretrability factor is calculated suposing that the system tunnels a barrier equal to $V$-. Q. The shell effects in the nuclear masses expressed in $Q$-value are reflected in the resulting barricr. Consequently, they are also included in the penetrability factor,

$$
P=\exp \left\{-\frac{2}{\hbar} \int_{\zeta 0}^{\zeta c} \sqrt{2 \mu[V(\zeta)-Q]} d \zeta\right\} .
$$

The action integral in the penetrability factor is given in terms of the variable of the model, $\zeta$, and the limits are the inser turring point:

$$
\zeta_{0}=R_{p}-\bar{R}_{1}
$$


and the outer one

$$
\zeta_{c}=\frac{Z_{1} Z_{2} e^{2}}{Q}
$$

Finally, the rate of the decay is calculated as

$$
\lambda=\lambda_{0} \mathcal{P}
$$

The characteristic time scale of surfuce oscilations in initial state of the system is used to define barrier assault frequency for the process, $\lambda_{0}$. Swiatecki ${ }^{i \varepsilon l}$ values of assault frequency with an odd-A parent nuclei hindrance effect,

$$
\lambda_{0}=\left\{\begin{array}{lll}
10^{20} & s^{-1} & \text {. A }=\text { odd } \\
10^{22} & s^{-1} & A=\text { even } .
\end{array}\right.
$$

were used in present work. With this frequency fixed, the half-life is promptly calculated,

$$
\tau=\frac{\ln 2}{\lambda}
$$




\section{Radii and Effective Mass}

The final radii of the fragments should be given by

$$
\tilde{R}_{i}=\sqrt[3]{\frac{Z_{i}}{Z_{p}}} R_{p}
$$

to be consistent with the uniform charge distribution considered in the Coulomb potential. The parent nucleus radius is determined by the simple formula

$$
R_{p}=r_{0} A_{p}^{i / 3}
$$

setting $r_{0}=1.37 \mathrm{fm}$ in all calculations.

With the Werner-Wheeler nipproximation for the velocity field of nuclear fluid in the prescission phase, we can obtain an expression for the kinetic energy of the system ${ }^{8,19}$. The constraint relations of eqs. $(1,2)$ and a constant radius for the cluster reduce the $k$ inetic energy expression to a quadratic form only in the velocity, $\dot{\zeta}$. From this expression we get the effective mass for the degree of freedom $\zeta$. In the center-of-mass frame we have for this effective mass,

$$
\begin{aligned}
m_{e f f}= & \sum_{i=1.2} \rho_{i}\left\{\frac{\left(z_{i}^{i}\right)^{2} v_{i}}{\pi}+2 \times(-1)^{i} z_{i}^{\prime} R_{i} R_{i}^{\prime}\left(R_{i}+d_{i}\right)^{2}\right. \\
& \left.+\frac{\left(R_{i} R_{i}^{\prime}\right)^{2}}{2}\left[\frac{4 R_{i}^{2}}{h_{i}}-9 R_{i}-7 d_{i}+12 R_{i} \ln \left(\frac{2 R_{i}}{h_{i}}\right)\right]\right\},
\end{aligned}
$$


where the mass density of auch spherical segment is $\rho_{i}=3 M_{i} /\left(4 \pi \bar{R}_{i}^{3}\right)$, and

$$
\begin{array}{ll}
z_{1}^{\prime}=-\frac{1}{2}\left[3 v_{2}+\pi a^{2}\left(R_{1}+d_{1}\right)\right] /\left(v_{1}+v_{2}\right) & z_{2}^{\prime}=z_{1}^{\prime}+1 \\
R_{1}^{\prime}=0 & R_{2}^{\prime}=-\frac{1}{2} \frac{h_{2}}{R_{2}} \\
a_{1}^{\prime}=\zeta-\xi & d_{2}=\xi \\
h_{1}=R_{1}-\zeta+\xi & h_{2}=R_{2}-\xi .
\end{array}
$$

The prime in the above expressions means the derivative with respect to the variable $\zeta$, and $v_{i}=\frac{\pi}{3}\left(R_{i}+d_{1}\right)^{2}\left(R_{i}+h_{i}\right)$ is the volume of each spherical segment. We have used this effective nass as the reduced mass $\mu$ in eq.10, defining the penetrability factor. Altbough the expression for the effective mass changes for difierent choices of coordinates and rrame the result for the decay rate is the same $\mathrm{e}^{[\mathrm{S} \mid}$. In fig. 3 we show the values of the effective mass of eq. 14 for different ciuster emission processes. We note that in the limit of asymptotic configurations, this expression for the effective mass reaches the reduced mass of the system when the fragments are already formed, $\bar{\mu}$, as it should be.

\section{Results and Discussions}

The calculated half-lives of different exotic decays observed in some secent experiments are shown in fig.4. The thich line of the upper region of the graph corresponds to the calculated result, and lise full circles are the logarithm of experimental data conpiled in ref.[16]. This result is for zero orbital angular momentum of the fragments. The coresponding reaction and values of half-lives are presented ia table 1 . In the table, the reactions with the same cluster emission in exotic process are grouped in blocks. The grougs are marked with broken and open arrows in the upper part of fig..

Whout ary tnodifotion in the nodel ong changing the inrut dato for masces, all

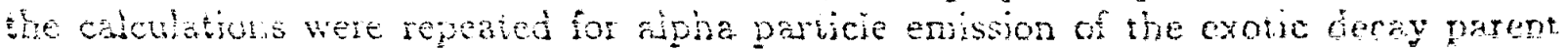
nucleus. The results for this essy are presented $b_{y}$ the thin line in the lover refion

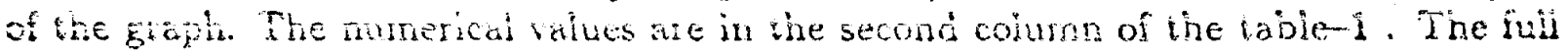


squares are the experimental results compiled in ref.[16]. For alpha decay it should be remembered that angular monentum is not completiy negligible for various decays and it might correct some small deviations from experimental data.

In fig. 5 we show how sensitive are our results to the effect of the centrifugal potential, including $V_{l}$ after the prescission point. We can see in fig.5-(a) that the effect is negligible for exotic decay, as it was pointed out before, but it may be significative to the alpha decay, as it is shown in fig.5-(b).

\section{Conclusions and Final Remarks}

We have analyzed the half-life for the exotic decay by using only the basic ingredient of the liquid drop fission theory. The Coulomb energy was calculated analyticaly for the moleculas phase of the sy'stem, with a double intersecting spheres parametrization for the deformed nuclear sysiem.

The Werner-Whecler approximation for the velocity field of nuclear fluid in the prescission phase defines the mass coefficient in the Gamow factor of the onedimensional barrier penetrability calculation.

The effective character of the model is marked by the surface tension defined in eq.(5). At this point we have note that nuclear radii are involved in this definition, so the nuclear sadius parameter $r_{0}$ controlls also the intensity of the surface potential. As we are not using explicitly effects of proximity force ${ }^{[15,18\}}$ to the potential, we have to compensate it with an appropriated intensity of our surface term. This fact justify our choise of $r_{0}=1.37 \mathrm{fm}$. Finally, it is important to remark that we fix this value for al! calculations and for both modes of decay presented in our results.

\section{Acknowledgement}

We are grateful for fruitful discussions with O. A. P. Tavares, C. E. Aguiar and E. L. Medeiros during the development of the present work. One of the authors, $M$. Gonçalves, would like to thank the partial support from CNPq. 


\section{Figure captions}

Figure 1: Shape parametrization of nuclear deformation. The nascent cluster corresponds to the splierical segment with radius $R_{1}$, and the heavier daugther is represented by the spherical segnent with radius $R_{2}$. The intersection of the spheres is a circle with radius $a$, and $\xi$ is the distance of the plane of the intersection to the geometrical center of the heavier frament. The distance between the geometrical centers of the fragments corresponds to $\zeta$.

Eigure 2: Onedimensional potential barrier. The coulomb energy is represented by the dotted curve and the long-dashed curve is the surface potential. Tho total potential is the continuous curve.

Eigure 3: Werner-Wheeler effective mass for differents cluster emission as a function of the distance between the geonerical certers of the spherical segments. After the scission point, the effective inass is constant and equal to the reduced mass of the system, $\bar{\mu}$.

Eigure 4: Hall-lives for the exotic and alphaccay. In the upper region of the graph the thick line is connecting the logarithms of half-life values for the exotic decays listed in table-1. The experimental data are shown by circles, and the arows atached to the first and last exotic decay data indicate that these values are only lower limits delermined experimentaily. The error bar for the other data are comparable to the circle size. The thin line in the lower region of the graph connects the results for logarithms of the alphe decay half-life of the parent nucle of the exotic decay. The data for the alfa decay hals-lives are shown by squares, with crror bar smaller than the square size.

Figure 5: Half-lives calculated whth the centhugal barrier. The solid line in part(a), represents the halt-life of exolic decays calculated with $=0$. The dotted lincs represent the half-life calculated with $\ell=6 \%$. In part-(b) the resuits for the sarne calculation to the alpha decay node of the exolic dacay parent nuclei are shown. 


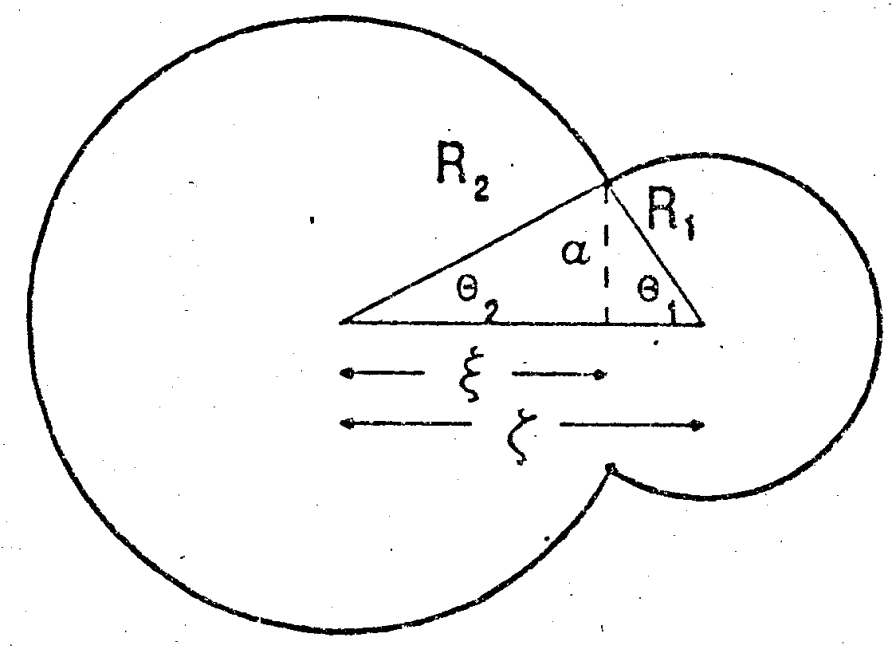

Figure 1 


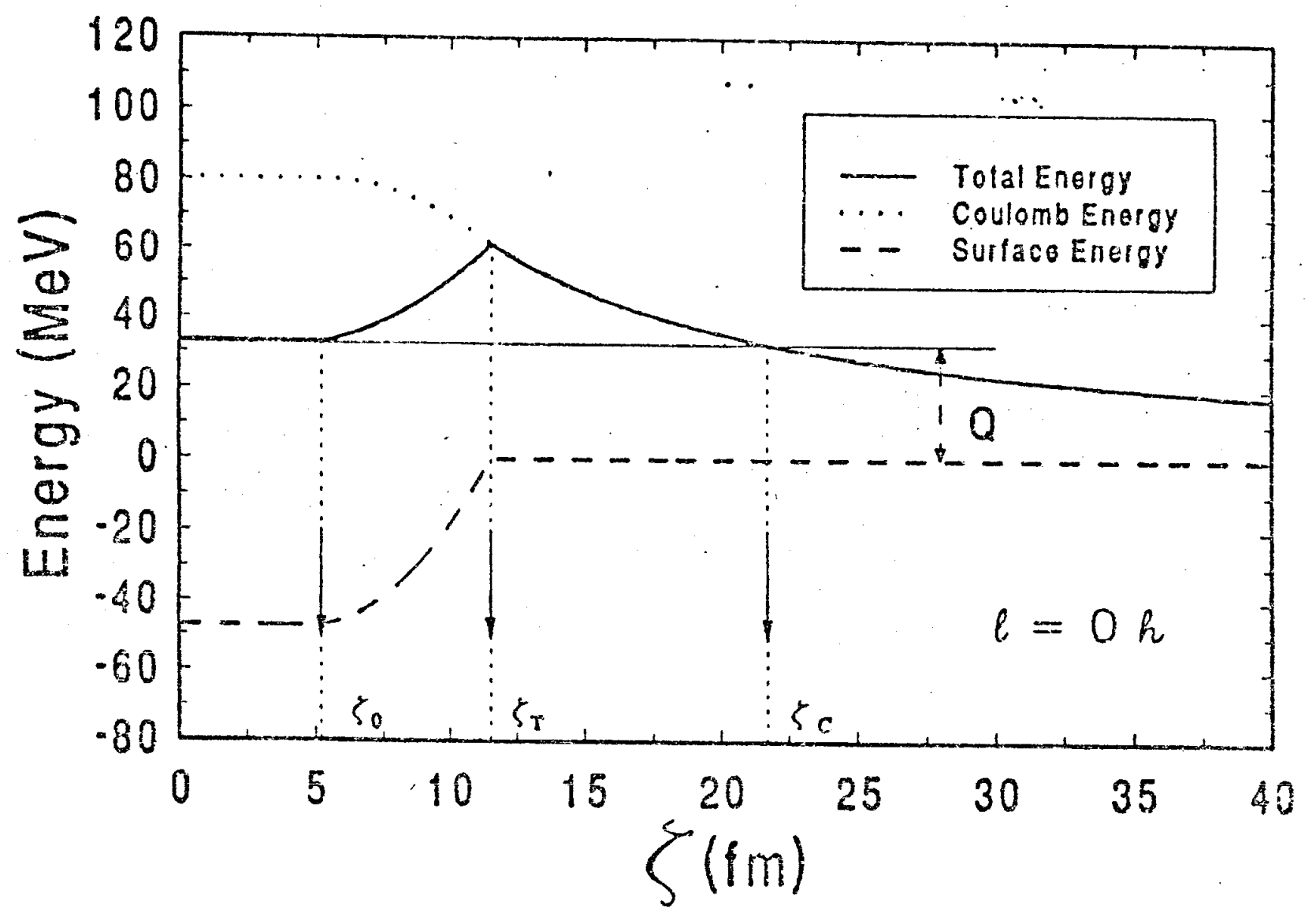

Figure 2 



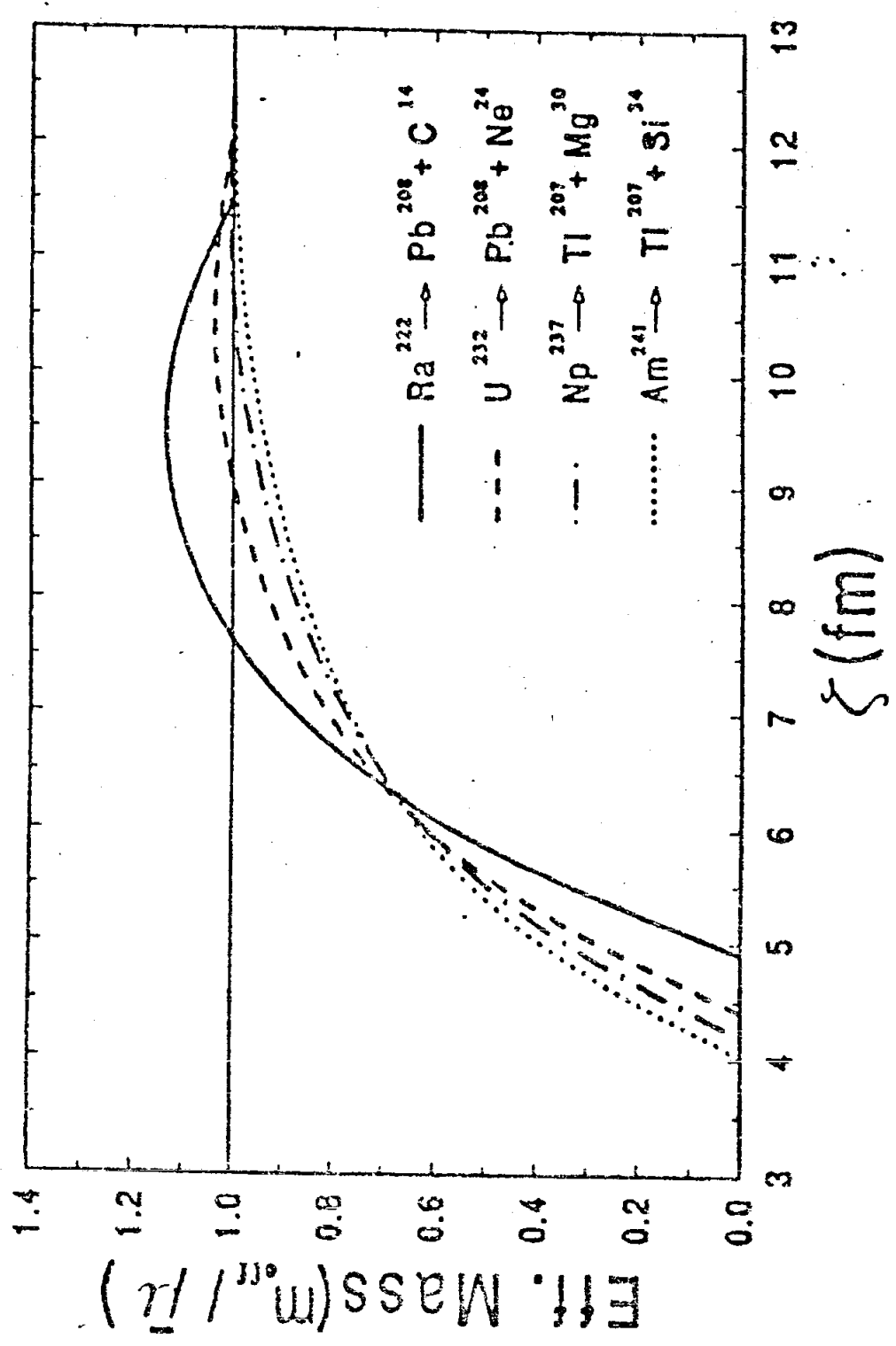

Eigure 3 


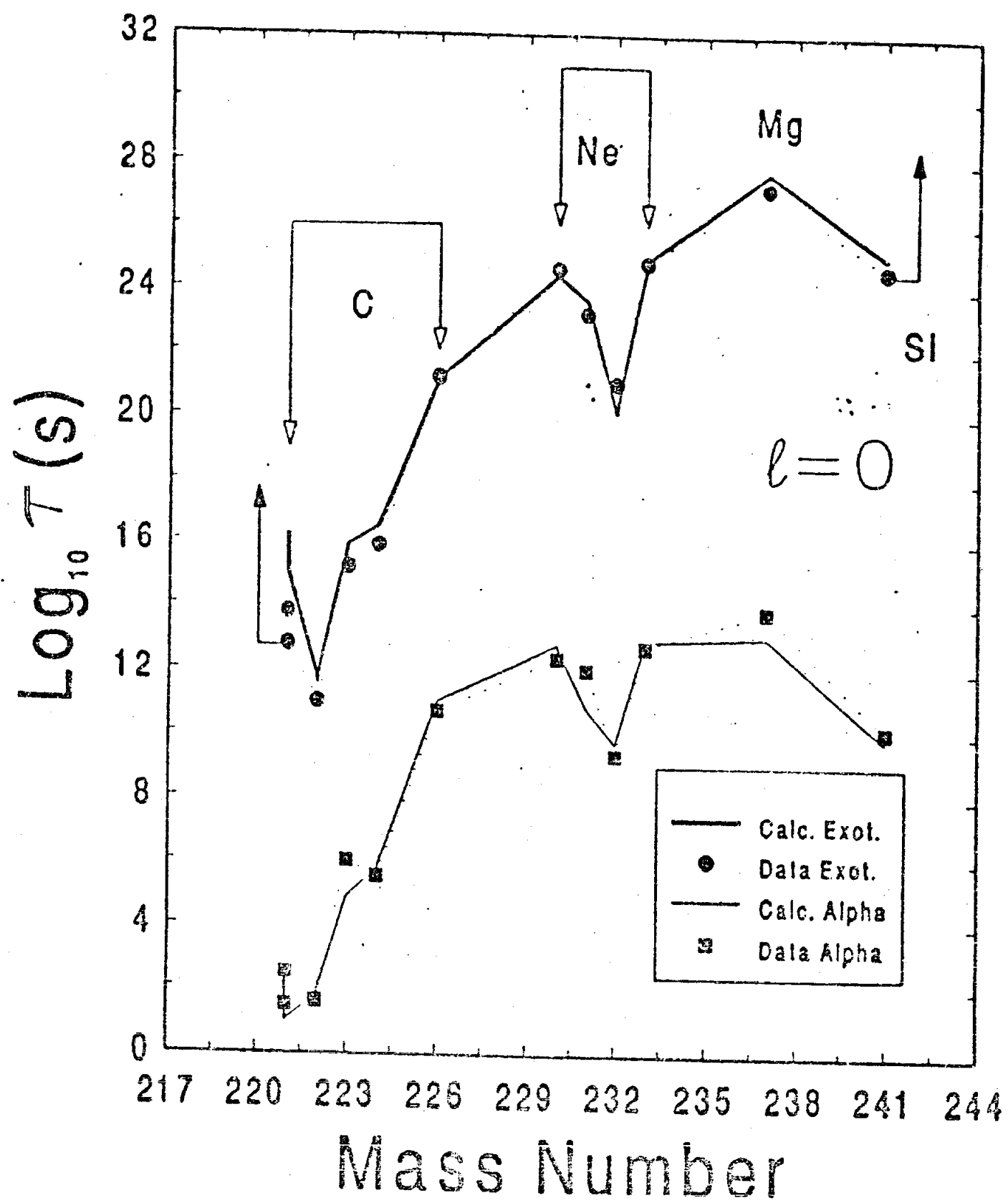

ligure 4 


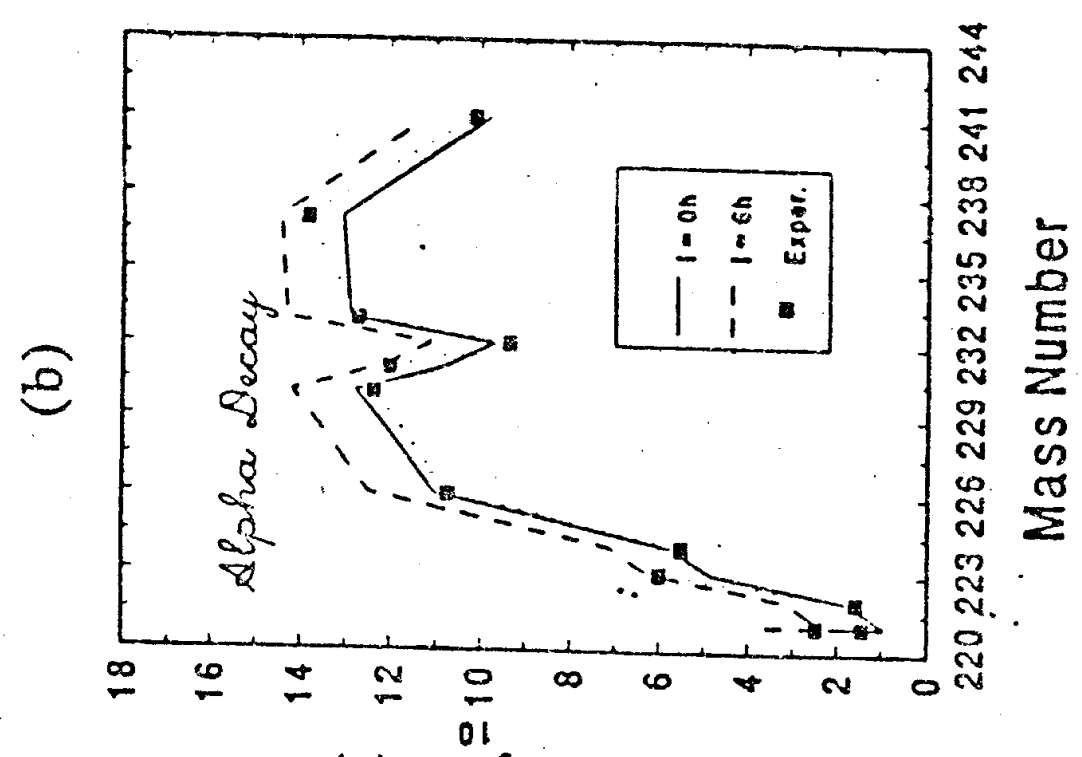

(s) $\perp 607$

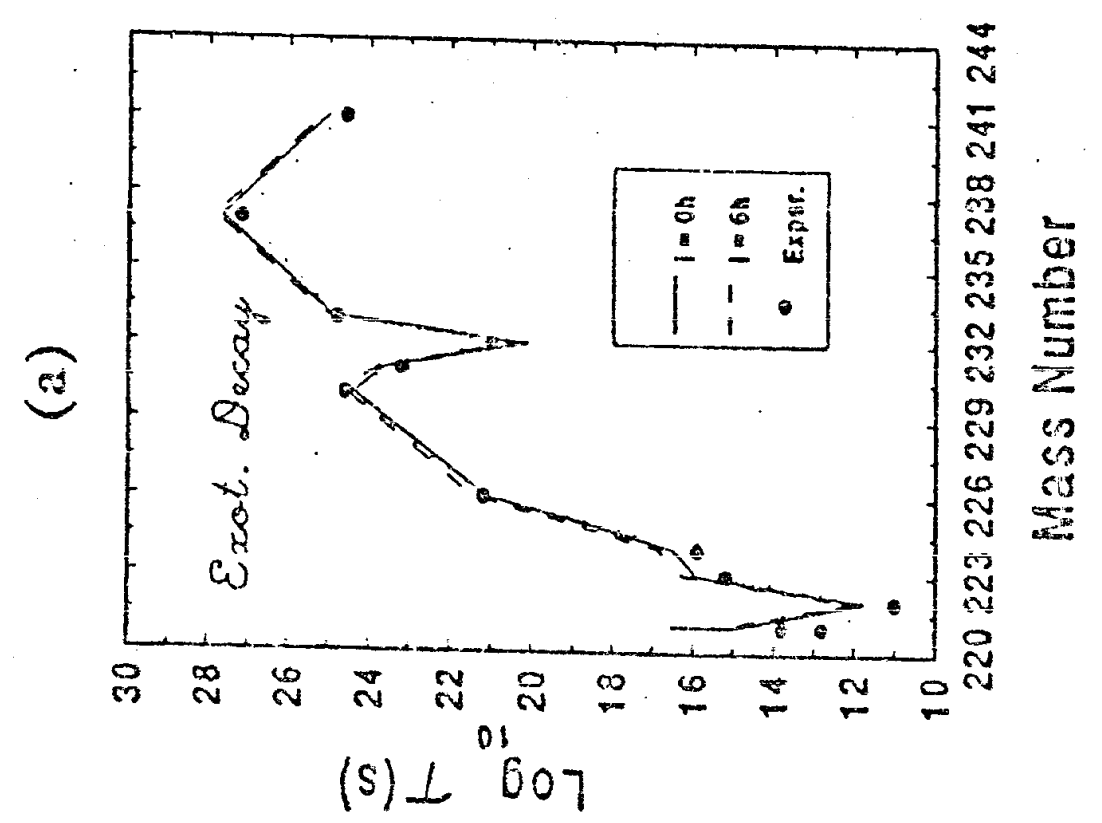

Figure 5 
Table and its caption

\begin{tabular}{|c|c|c|}
\hline Decay Reaction & $\log _{10}{ }^{E x o t}(s)$ & $\log _{10} \tau^{\alpha}(s)$ \\
\hline $\mathrm{Fr}^{221} \rightarrow \mathrm{C}^{14}+\mathrm{Tl}^{207}$ & 16.21 & 2.21 \\
$\mathrm{Ra}^{221} \rightarrow \mathrm{C}^{14}+\mathrm{Pb}^{207}$ & 14.98 & 0.95 \\
$\mathrm{Ra}^{222} \rightarrow \mathrm{C}^{14}+\mathrm{Pb}^{208}$ & 11.72 & 1.73 \\
$\mathrm{Ra}^{223} \rightarrow \mathrm{C}^{14}+\mathrm{Pb}^{209}$ & 15.82 & 4.80 \\
$\mathrm{Ra}^{224} \rightarrow \mathrm{C}^{14}+\mathrm{Pb}^{210}$ & 16.50 & 5.73 \\
$\mathrm{Ra}^{226} \rightarrow \mathrm{C}^{14}+\mathrm{Pb}^{212}$ & 21.09 & 11.02 \\
\hline $\mathrm{Th}^{230} \rightarrow \mathrm{Ne}^{24}+\mathrm{Hg}^{206}$ & 24.39 & 12.76 \\
$\mathrm{~Pa}^{231} \rightarrow \mathrm{Ne}^{24}+\mathrm{Tl}^{207}$ & 23.60 & 10.80 \\
$\mathrm{U}^{232} \rightarrow \mathrm{Ne}^{24}+\mathrm{Pb}^{208}$ & 20.10 & 9.70 \\
$\mathrm{U}^{233} \rightarrow \mathrm{Ne}^{24}+\mathrm{Pb}^{209}$ & 24.73 & 12.89 \\
$\mathrm{U}^{233} \rightarrow \mathrm{Ne}^{25}+\mathrm{Pb}^{208}$ & 24.88 & 12.89 \\
\hline $\mathrm{Np}^{237} \rightarrow \mathrm{Ng}^{30}+\mathrm{Tl}^{207}$ & 27.68 & 13.05 \\
\hline $\mathrm{Am}^{241} \rightarrow \mathrm{Si}^{34}+\mathrm{Tl}^{207}$ & 25.01 & 9.79 \\
\hline
\end{tabular}

Table 1: Decay reaction and calculated half-lives. The first mumerical column is the logarithm of the exotic decay half-life, and the second one corresponds to the logaribm of the alpha decay half-live of the exotic decay parent nuclei. 


\section{References}

(1) H. G. de Cartaho, J. B. Martins, 1. O. de Souza, and O. A. P. Tavares, An. Acad. Bras. Ciènc. 47, $567(1905)$

[2] I. O. de Sonza, M. S. Hesis, Centro Brazileiro de Pesquisas Físicas, 1975.

[3] O. A. P. Tavares, Doctora thesis, Centro Brasileiro de Pesquisas Físicas, 1978.

[4] H. J. Rose and G. A. Jones, Nature 307, 245 (1984).

(5] D. V. Alessandrov, A. E. Belyatskii, Yu. A. Glukhov, E. Yu. Nikal'skii, E. V. Novatskii, A. A. Oglobin, D. N. Stepanov, JETP Lett., 40, 909 (1984).

[6] D. N. Poenaru, M. Ivascu, D. Mazuilu, Comp. Phys. Commun. 19, 205 (1980).

(7) D. N. Poenaru, M. Ivascu, J. Physique 45, 1099 (1984).

[8] Pic-Pichac G. A., Sov. J. Nucl. Phys 44(6), 923 (1986).

[9] E. Hourani, M. Hussonnois, D. N. Poenaru, Ann. Phys. Er., 14, 311 (1989).

[10] D. N. Poenaru, W. Greiner, M. Ivascu, D. Mazilu,I. H. Plonski, Z. Phys. A-Atora Nuclei 325,435 (1950).

[11] G. Shanmugam and D. Kanalaharan, Phys. Rev. C 41, 1742 (1990).

[12] M. Gaudin, Le Journal de Physique, 35885 (1974).

[13] G. Gamow, Z. Phys. 51, 204 (1928).

[14] B. Buck, A. C. Marchant, S. M. Perez, Nucl. Phys. A 512, 483 (1990).

[15] A. K. Wapstra and G. Audi, Nucl. Phys. A 432, 1 (1985).

[16] Yi-Jin Shi and W. J. Swiatechi, Phys. Rev. Lett, 54, 300 (1985).

[17] H. G. de Carvalho, J. D. Martins, O. A. B. Tavares, Phys. Rev. C 34, 2201 (1986),

[18] Yi-Jin Shi, and W. J. Swiatecki, Nucl. Phys. A fos, 205 (1087).

[10j D. N. Poenaru, J. A. Mazuhn, W. Greiner, M. Ivascu, D. Mazilu, I. Ivascu, Z. Phys. A-Atonic Nuclei 333, 291 (1989). 
